\title{
Research on Integrated Framework and Realization Model of EIS
}

\author{
Fangxiao WANG ${ }^{1, a}$, Chenlu DING ${ }^{2, ~ b}, \mathrm{Hao}_{\mathrm{YAN}}^{3, \mathrm{c}}$
}

${ }^{1}$ Nanjing Telecommunication Technology Institute, Nanjing, 210007, China

${ }^{2}$ Nanjing Telecommunication Technology Institute, Nanjing, 210007, China

${ }^{3}$ Nanjing Telecommunication Technology Institute, Nanjing, 210007, China

aemail: wangfx0123@163.com, bemail:dingchenlu1030@163.com, 'email:yanhao0114@yeah.net

Keywords: Integrated Framework; Component Integration; Service Integration

\begin{abstract}
The paper analyses the construction model of enterprise information systems (EIS), and puts forward a layering and service-oriented integrated framework. The paper expounds that the major function and realization model from four aspects of data, component, service, and portal integration, which provides an effective and theoretical direction for establishing an integrative environment of development, integration and operation.
\end{abstract}

\section{Introduction}

In recent years, there is a great improvement of capabilities of enterprise information systems (EIS), which is becoming a highly efficient organization and management method in enterprises. These systems play an important role in enterprise operations such as normalizing business process and increasing working efficiency. Data and information is the key to enterprise systems because the operation and management of enterprises can hardly run without its support. However, these autonomous and in many cases heterogeneous systems are historically not designed to collaborate with other applications, as sales managers have tended to develop their systems independently and without any co-ordination. There is also the complexity of existing information systems, which in many cases have fixed and rigid structures for messages, interfaces and databases. It may result in a lack of system interconnection and utilization of information resources and data resources. Therefore, how to integrate various heterogeneous systems with recent systems and establish an effective integrated framework is becoming a big challenge for various enterprises.

The methodological context of enterprise application integration (EAI) with its domain engineering and application engineering techniques dominates development and integration solutions. The terminology already points to architecture as the key to a solution for the integration problem[1]. To achieve the construction goal including unified portal, integrated service, integrated component and integrated data, this paper analyzes the construction model of the enterprise information system and proposes a hierarchical and service-oriented integrated framework which can be used universally. And the paper expounds that the major functions and realization modes from four aspects, which provides an effective and theoretical direction for establishing an integrative environment of development, integration and operation.

\section{Construction Model of EIS}

According to the departments' functions and operating modes, the construction model of EIS is established by the top-down design ideas. This construction model consists of application system, business systems, functional components and data sources. Figure 1 shows the construction model of EIS. 


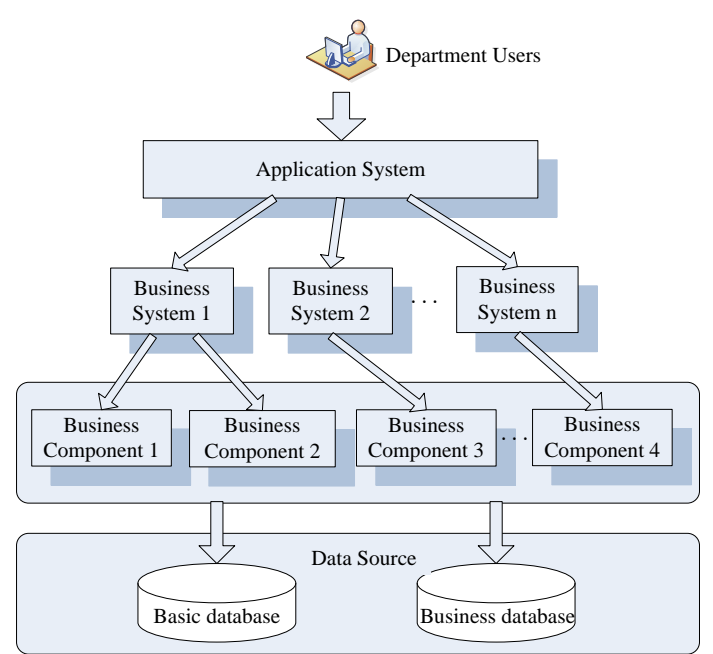

Fig.1. Construction Model of EIS

Application systems: The application system provides a unified portal for the ultimate department users to deal with their business. With the method of integration and custom, the application systems used in different departments are integrated by one or several business subsystems.

Business systems: There are two types of business systems. One type is the systems which have independent business functions, such as sales system, inventory system, and logistics system and so on. The other type is the systems which have general functions, such as document processing system. The application system is integrated by these business systems through the technical means including data migration, function selection, procedure customization, system deployment, etc.

Business components: Enterprise IT architectures consist of complex systems of interdependent components that are governed by business processes[2].The business component is usually the software module that provides single business functions, such as the sales statistics and the inventory information analysis. On the other hand, it may be the module having common functions like the document processing.

Data source: Data source is the data resource which can be accessed by all kinds of business components. It is mainly divided into two groups: basic database and business database. The basic database which including of the core basic data, the business basic data and the data dictionary, is the standard data storage area created in order to achieve the information sharing and data exchanging vertically and laterally. Meanwhile, the business databases cover different business fields such as sales business, inventory management and logistics management.

\section{Research on Integrated Framework}

\subsection{Design Principles}

The framework makes use of software toolkits to integrate external applications, thus becoming a robust, friendly-used and stable integration platform. To sum up, the framework design should have the following principles:

(1) Sustainability. Above all, in the design an upgraded and sustained architecture should be constructed. Moreover, the integration of legacy systems should be fully considered, that is to say, it should offer extendible, flexible and compatible solutions to the integration of legacy systems so that it reaches to the effect of "the plug and play".

(2) Extensibility. It is should be avoid constructing a tight coupling framework centering on the centralized database. With regard to interface design, the interface integrity is very important because the new systems and the new functions need to be added conveniently. At the same time, the operation of other systems should be not affected by the technical reconstruction and function expansion of the legacy systems. 
(3) Reusability. Based on the loose coupling designing idea, the framework should be designed to reduce the complexity of the single business function through a flexible and service-oriented composing mode. To limit the influence which is brought by a change of one function to the other can not only improve the reusability to fit in with the business variability, but also reduce the risk or the cost of functional changes.

\subsection{Framework Design}

As shown in Figure 2, the integrated framework is proposed based on the above principles. The framework includes four parts: data integration, component integration, service integration and portal integration. Every part of the framework includes several services which could be composed and executed for integrated activities. The basic concept, main functions, and realization model of every part of the integrated framework will be described in detail next.

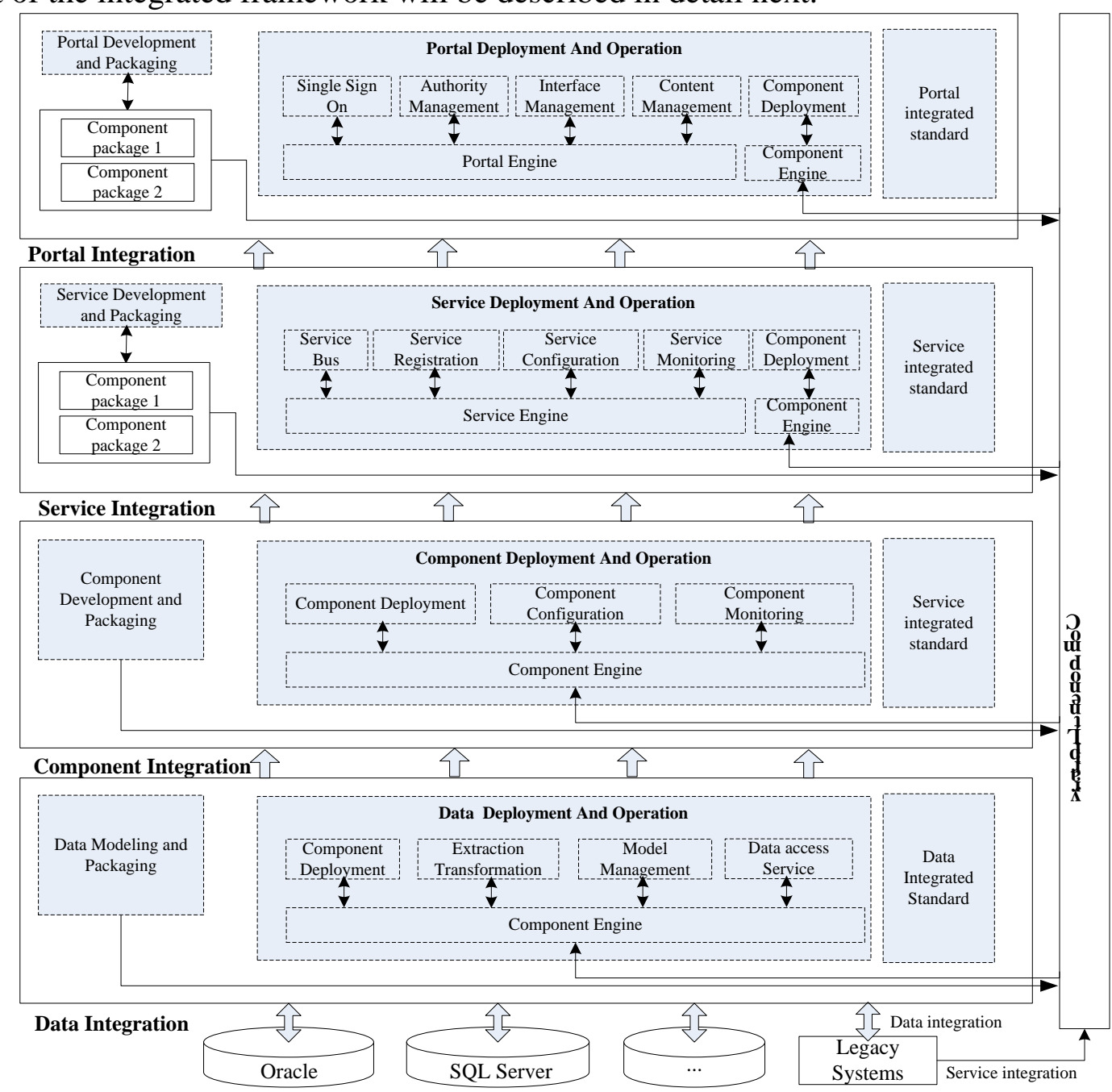

Fig.2. Integrated Framework of EIS

\subsubsection{Data Integration}

Data integration focuses on providing data entities for business function components to access business data sources. It is the basis of component integration and service integration emphasize solve the access and integration problems existing between new database and other legacy databases. Data integration includes of data modeling and packaging, data deployment and operation, data integrated standards and so on. Using data modeling and packaging, metadata and initial data in databases is fetched and packaged into compressed files which can be deployed. Using data deployment and operation, the packaged data components are arranged to the named database servers, and the components can be installed, deployed, upgraded and unloaded. Data deployment is better viewed as an infrastructure to provide a unified data access interface; to 
support the development and running of business software based on SOA; to support the access and operation to mostly relational databases like Oracle and SQL Server.

The realization model of data integration is as follows:

(1) Data Modeling and Packaging: It is used to establish business database, and check the running environment, relationship among components, interface calling of data components according to integrated principles. After being packaged, the data components are submitted to system manager by data deployment and operation.

(2) Data Components Management and Allotment: It is used to put the packaged data components to the components library, extract them which need to be allotted from the library by system manager, and then send them to the relevant departments. The data component has been received is submitted to the components library of each department. Then system manager can make a series of operations such as examination and approval, classification, and analysis, etc.

(3) Data Components Deployment: It is used to make a centralized data deployment. On the basis of business requirement of each department, the system manager utilizes these received data components to establish the applied database through the components deployment toolkit.

(4) Data Access Service: Data integration platform designed to build data integration package for different data sources[3]. Data access service provides the unified data access for components. According to the need of software development, the mapping relationship between data objects and tables in database are deployed. And then, to complete the database access through operating data objects by using the object access language.

(5) Legacy Systems Data Integration: It is usually used when there are a lot of difficulties in service integration. The first step is to analyze the database structure and standard of legacy systems in accordance with the technical system and standard specification of information exchange and sharing. The second step is to integrate the data of legacy systems by the methods including data extracting, data conversion, data migration, data synchronization and data components packaging and deployment.

\subsubsection{Component Integration}

Component integration is the most important part of the integration framework. Component is that a software package which has been encapsulated, interface-defined, and could be deployed independently and released[4]. That is to say, component is a composed unit which has interface functions. Component integration provides the functions including component developing and encapsulating, component deployment and operation, and standard integration principles.

The realization model of component integration is as follows:

(1) Component development and packaging: There are two steps. The first step is to develop functional component following component integration standards, especially on the aspects of running environment, relationship among components, style of interface, and method of interface calling and so on. The second step is to package the components and submit them to system manager by using the component packaging tool.

(2) Component management and distribution: The developed components are stored in the components library in a unified manner. When the components are needed, system manager extract them from the library and send them to the related departments. In each department, the received components are submitted to its own components library. System manager can make a series of operations such as examination and approval, classification, and analysis on the library.

(3) Assembly and deployment of system application: According to the business demand of each department, system manager choose the needed functional components and make them into system component packages using the assembly tool. System deploying personnel use the component deployment tool to centralized deploy the system component packages, finally forms to a performable business system.

(4) System running and monitoring: System users directly operate the business software on IE. They can complete all of business work through choosing the needed business functions on the pages. At the same time, system manager can monitor the system application and the functional 
components, such as the called components, the called frequentness, the duration of a single call, etc.

\subsubsection{Service Integration}

Service integration is the core of the integration framework. Not only the manifestation of service is components, but also service running and deployment relies heavily on component integration. All of resources related to service integration are based on every component. Business components are deployed to business system which has relatively independent business functions through the method of service integration. It provides the functions including service development and packaging, service deployment and operation, and standard integration principles.

The realization model of service integration is as follows:

(1) Service planning and design: According to the actual business demand, the being fetched services are analyzed and screened. The service design tool provides visualization editor, visualization view and guide design service. Such as business description, service interface, service level requirement are the main part of service design.

(2) Service assembly and arranging: It is the two main forms of service reuse. Service assembly is to combine one or a lot of business components through assembling and to release them to service. Service arranging is to arrange one or a lot of services to form a new service, and moreover, many of single service could be arranged to a unified compound service which could meet the requirement on the higher application layer[5]. On the layer of business system, we can use business logic to find and call the related service from the service registration center. Then, according the real business workflow, we can run a service arrange to form a business workflow and define its relative information. When business logic is changed, we can do only is to adjust the service arranging and call the relative service. That not only can make the business system flexibly and quickly adapt to the business operation, but also can fully embody the reusability of service[6].

(3) Service registration and operation: The needed services are registered on service bus by system manager. Considering the business requirement and the actual environment of each department, system manger can deploy the services accordingly. Service engine provide engine support for service loading, analysis and running, and have the core capabilities such as arrangement, registration, analysis, run, and supervisory control etc. At the same time, system manager can monitor and gain the current situation when system is calling services by the service monitor tool. Such as the called components, the called frequentness, and the duration of a single call could be monitored by system manger.

(4) Legacy Systems Service Integration: The first step is to analyze the existing functions of legacy systems. In accordance with the technical system and standard specification of information exchange and sharing, the second step is to make service integration on legacy systems by the integrating methods including service development, service registration and release, service engine.

\subsubsection{Portal Integration}

Portal integration is the top layer of the framework, which provides users with a unified entrance to application system. In order to ensure the information safety, after single-point logging on and being given corresponding permission, system users can be provided personalized services. Portal integration provides the functions including portal development and packaging, portal deployment and operation, and standard integration principles.

The realization model of service integration is as follows:

(1) Portal Development and Packaging: Firstly, the portal framework, pages and components should be developed according to the practical requirements, and the components should be packaged within the standard integration principles. Then, after the software being developed, the packaged components should be submitted to system manager through the system managing tool.

(2) Portal Deployment and Operation: It is the key factor that determines the success of portal integration. System manager should maintenance the information of all the departments and personnel. And then, they should be allocated the suitable authority to guarantee them to access the portal application correctly and safely. Moreover, users can have an individual customization on the 
portal application.

\section{Conclusion}

Enterprise application integration is a problem that has dominated the enterprise wide view on IT architectures and software applications for a long time. With the emergence of these problems such as the weak capability of system interconnection and lacking of information sharing effectively, to construct an incorporate integration framework is necessary. Based on the principles of sustainability, extensibility and reusability, the integration framework which have data integration, component integration, service integration, and portal integration is proposed in this paper. In this framework, the approach deals with some integration issues such as: data access, component development and packaging, service registration and operation, legacy systems integration and portal deployment. This paper has provided a practical solution which can be used for reference for the EIS design and development staff.

\section{References}

[1] S. Conrad, W.Hasselbring, A.Koschel, and R.Tritsch, Enterprise Application Integration, Elsevier, Spektrum, 2006.

[2] F.Leymann, W.Reisig, S.R.Thatte, and W.M.P. van der Aalst, "The role of business processes in service oriented architectures”, Dagstuhl Seminar Proceedings, Vol.06291, 2006, http://drops.dagstuhl.de/opus/volltexte/2006/832/pdf/06291_abstracts_collection.832.pdf.

[3] Ying Wang, Daoping Wang, Guangli Liu, Di Li. Data Integration Platform for Village Emergency, International Journal of Digital Content Technology and its Applications Volume 4, Number 4, July 2010.

[4] Zuyu Yu, Xiaofei Yao, Yongqian Ai. Application System Based on Component Technique [J]. Command Information System and Technology, 2012,3(6):58〜61.

[5] Hefeng Zheng, Siju Chen. Research on military information system integration based on SOA [J]. Fire Control and Command Control, 2010, 35(1):81 83.

[6] Wei-xing WANG, Chen-guang WANG. Integration Framework of Enterprise Information System based on Service-Oriented Architecture[J]. Computer Engineering, 2010， 36(18): 29 31. 